\title{
Magnetic targeting of paclitaxel-loaded poly(lactic- co-glycolic acid)-based nanoparticles for the treatment of glioblastoma
}

This article was published in the following Dove Press journal:

International Journal of Nanomedicine

\author{
Lakshmi Pallavi Ganipineni' \\ Bernard Ucakar' \\ Nicolas Joudiou ${ }^{2}$ \\ John Bianco' \\ Pierre Danhier ${ }^{2}$ \\ Mengnan Zhao' \\ Chiara Bastiancich' \\ Bernard Gallez ${ }^{2}$ \\ Fabienne Danhier ${ }^{1, *}$ \\ Véronique Préat ${ }^{1, *}$
}

'Université catholique de Louvain, Advanced Drug Delivery and Biomaterials Research Group, Louvain Drug Research Institute, Brussels, Belgium; ${ }^{2}$ Université catholique de Louvain, Louvain Drug Research Institute, NEST Nuclear and Electron Spin Technologies Platform, Brussels, Belgium

*These authors contributed equally to this work

\begin{abstract}
Introduction: Glioblastoma (GBM) therapy is highly challenging, as the tumors are very aggressive due to infiltration into the surrounding normal brain tissue. Even a combination of the available therapeutic regimens may not debulk the tumor completely. GBM tumors are also known for recurrence, resulting in survival rates averaging $<18$ months. In addition, systemic chemotherapy for GBM has been challenged for its minimal desired therapeutic effects and unwanted side effects.
\end{abstract}

Purpose: We hypothesized that paclitaxel (PTX) and superparamagnetic iron oxide (SPIO)loaded PEGylated poly(lactic-co-glycolic acid) (PLGA)-based nanoparticles (NPs; PTX/SPIO$\mathrm{NPs}$ ) can serve as an effective nanocarrier system for magnetic targeting purposes, and we aimed to demonstrate the therapeutic efficacy of this system in an orthotopic murine GBM model.

Materials and methods: PTX/SPIO-NPs were prepared by emulsion-diffusion-evaporation method and characterized for physicochemical properties. In vitro cellular uptake of PTX/SPIONPs was evaluated by fluorescence microscopy and Prussian blue staining. Orthotopic U87MG tumor model was used to evaluate blood-brain barrier disruption using $\mathrm{T}_{1}$ contrast agent, ex vivo biodistribution, in vivo toxicity and in vivo antitumor efficacy of PTX/SPIO-NPs.

Results: PTX/SPIO-NPs were in the size of $250 \mathrm{~nm}$ with negative zeta potential. Qualitative cellular uptake studies showed that the internalization of NPs was concentration dependent. Through magnetic resonance imaging, we observed that the blood-brain barrier was disrupted in the GBM area. An ex vivo biodistribution study showed enhanced accumulation of NPs in the brain of GBM-bearing mice with magnetic targeting. Short-term in vivo safety evaluation showed that the NPs did not induce any systemic toxicity compared with Taxol ${ }^{\circledR}$ (PTX). When tested for in vivo efficacy, the magnetic targeting treatment significantly prolonged the median survival time compared with the passive targeting and control treatments.

Conclusion: Overall, PTX/SPIO-NPs with magnetic targeting could be considered as an effective anticancer targeting strategy for GBM chemotherapy.

Keywords: nanomedicine, glioblastoma, magnetic targeting, nanoparticles, PLGA-based nanoparticles

\section{Introduction}

Glioblastoma (GBM) is one of the most aggressive tumors occurring in the central Correspondence: Véronique Préat Louvain Drug Research Institute, Université catholique de Louvain, Avenue Mounier 73, Boîte bl.73I2, 1200 Brussels, Belgium

Tel +3227647309

Fax +32 27647398

Email veronique.preat@uclouvain.be nervous system. The incidence rate of GBM is $\sim 17 \%$ of total central nervous system cancers. ${ }^{1}$ Approximately $70 \%$ of GBM patients will experience disease progression within 1 year of diagnosis, with $<5 \%$ of patients surviving 5 years after diagnosis. Aggressiveness of GBM is characterized by highly proliferating infiltrative cells, indistinct tumor margins, high intra- and intertumor heterogeneity, peritumoral edema 
and inflammation. ${ }^{2}$ Therapy for GBM is very challenging, as the tumors are very aggressive due to infiltration into the surrounding normal brain tissue and are highly recurrent. Even a combination of the available therapeutic regimens (chemotherapy, radiotherapy and surgical resection) may not debulk the tumor completely and results in very low survival rate $<18$ months. Furthermore, systemic chemotherapy for GBM treatment is more challenging for its minimal desired therapeutic effects due to biological barriers such as bloodbrain barrier (BBB) and unwanted distribution in healthy tissues leading to systemic toxicity. ${ }^{3}$

Nanomedicine is an alternative approach to address the conventional chemotherapy drug delivery challenges. An ideal therapy for GBM would be a multifunctional system that not only avoids the invasiveness/incompleteness of conventional surgeries but also mitigates the setbacks of chemo- and radiotherapies. Several multifunctional nanocarrier systems such as nanotheranostics are under investigation to address the unmet clinical needs of GBM patients. ${ }^{4-6}$

Noninvasive "magnetic targeting", which can be achieved by the application of an external magnetic field, may synergize with the already existing enhanced permeability and retention (EPR) effect of nanocarriers and thereby enhance the therapeutic indices. Superparamagnetic iron oxide nanoparticles (SPIO-NPs), which are used as a $\mathrm{T}_{2}$ contrast agent for magnetic resonance imaging (MRI), are being investigated in combination with anticancer drugs for GBM therapy. ${ }^{7}$ SPIO-NPs are reportedly associated with minimal-to-no toxicity and are well known for their magnetic properties and as an MRI diagnostic agent. ${ }^{8}$

Paclitaxel (PTX) is a powerful anticancer drug that is often recommended as a first-line chemotherapeutic agent against many kinds of cancers. It acts by stabilizing microtubules during cell division and leads to the interruption of mitosis. ${ }^{9}$ Even though PTX is a highly potent agent, its use in clinical treatment for GBM is limited by failure to reach the tumor site in sufficient concentrations due to the BBB. PTX has also been associated with drug resistance as well as other side effects. Several reports have shown that PTX encapsulated in nanocarriers can cross a disrupted or leaky BBB, which is a common feature associated with GBM. ${ }^{10-12}$

Previously, our group has demonstrated the therapeutic and diagnostic efficacy of nanotheranostic particles along with different targeting strategies using PEGylated poly(lactic-coglycolic acid) (PLGA)-based nanoparticles (NPs) in subcutaneous CT26 colon carcinoma. ${ }^{13,14}$ PEGylation hinders opsonization of the NPs. Moreover, PLGA is biocompatible and biodegradable and is approved by regulatory agencies as an excipient for parenteral products.

In the current study, we aimed to evaluate the efficacy of NPs and different targeting strategies in an orthotopic GBM model. We hypothesized that the PTX/superparamagnetic iron oxide (SPIO)-loaded PEGylated PLGA-based NPs (PTX/SPIO-NPs) can serve as an effective nanocarrier for magnetic targeting purposes and can demonstrate their therapeutic efficacy in orthotopic GBM-bearing mice.

\section{Materials and methods \\ Materials}

PTX was purchased from Chemieliva Pharmaceutical Co. (Chongqing, China). PLGA (lactide/glycolide molar ratio of 50:50, molecular weight $[\mathrm{MW}]=7,000-17,000)$, iron(II) chloride, iron(III) chloride, oleic acid, sodium hydroxide, hydrochloric acid, DAPI, MTT, polyvinyl alcohol (PVA; MW = 30-70 $\mathrm{kDa})$, potassium hexacyanoferrate(II) trihydrate, xylazine hydrochloride and American Cancer Society reagent $(98.52 \%-102.0 \%)$ were purchased from SigmaAldrich Co. (St Louis, MO, USA). Poly(caprolactone)- $b$ PEG (PLGA- $b$-PEG) (MW = 10,040-4,600), PCL- $b$-PEG $(\mathrm{MW}=13,100-5,000)$ and fluorescein isothiocyanate (FITC)-labeled PLGA were synthesized and characterized as previously described. 9,15,16 U87MG cells (American Type Culture Collection [ATCC ${ }^{\circledR}$ ], Manassas, VA, USA, HTB-14 ${ }^{\mathrm{TM}}$ ) and Eagle's Minimum Essential Medium (EMEM) were obtained from ATCC. Fetal bovine serum (FBS), trypsin-EDTA and penicillin-streptomycin mixtures were obtained from Thermo Fisher Scientific (Waltham, MA, USA). Poly-D-lysine hydrobromide (MW = 70,000150,000) was obtained from MP Biomedicals (Eschwege, Germany); Nimatek (ketamine $100 \mathrm{mg} / \mathrm{mL}$ ) was obtained from Eurovet Animal Health B.V. (Bladel, the Netherlands); gadoterate meglumine (Gd-DOTA; Dotarem ${ }^{\circledR}$ ) was obtained from s.a. Guerbet n.v. (Brussels, Belgium) and formaldehyde solution was purchased from EMD Millipore (Billerica, MA, USA; 4\%). All other chemicals and solvents used were of analytical grade.

\section{Formulation and physiochemical characterization \\ Preparation of PTX and SPIO-loaded NPs (PTX/SPIO-NPs)}

Hydrophobic SPIO coated with oleic acid was synthesized at $80^{\circ} \mathrm{C}$ under an inert atmosphere using a coprecipitation method of ferrous and ferric salts in an alkaline medium 
following the procedures as previously described. ${ }^{13,14,17}$ PTX/SPIO-NPs were prepared by an emulsion-diffusionevaporation method ${ }^{9,13,14,16}$ with minor modifications. Briefly, PTX (2.5 mg), PLGA (70 mg), PCL- $b$-PEG $(15 \mathrm{mg})$ and PLGA- $b$-PEG $(15 \mathrm{mg})$ were dissolved in dichloromethane containing SPIO $(7.7 \mathrm{mg} / \mathrm{mL}$ iron [Fe] concentration). The mixture was added to 3\% PVA aqueous solution, vortexed and emulsified at $50 \mathrm{~W}$ using an ultrasonicator $(3 \times 30 \mathrm{~s})$. The generated emulsion was added dropwise to a $1 \%$ PVA aqueous solution under magnetic stirring at $400 \mathrm{rpm}$, and the organic solvent was allowed to evaporate overnight. The suspension was filtered $(1.2 \mu \mathrm{m})$ to remove any possible aggregates. The filtrate was resuspended in $15 \mathrm{~mL}$ of water and ultracentrifuged three times at $15,000 \mathrm{rpm}$ for $30 \mathrm{~min}$ at $4^{\circ} \mathrm{C}$. After the last ultracentrifugation, NPs were suspended in $1 \mathrm{~mL}$ of water. The SPIO-NPs and PTX-loaded NPs (PTX-NPs) were prepared using the same protocol. Polysorbate 80 (PS80) coating of NPs was performed by suspending NPs in water with $1 \%$ surfactant solution and incubated for $30 \mathrm{~min}$ at ambient temperature. ${ }^{18}$ Fluorescently labeled PLGA-based NPs (PLGA-NPs) were prepared similarly using PLGA covalently labeled with FITC. ${ }^{15}$

\section{Physiochemical characterization of PTX/SPIO-NPs}

The Fe concentration of SPIO was measured by electron spin resonance (ESR) spectroscopy (Bruker Optik GmbH, Ettlingen, Germany) and validated by inductively coupled plasma mass spectrometry (ICP-MS) using an Agilent 7500ce instrument (Agilent Technologies, Santa Clara, CA, USA). ${ }^{19}$ The particle size and polydispersity index (PDI) of the NPs were measured by dynamic light scattering, and the zeta potential was determined by laser Doppler velocimetry using a NanoSizer Zeta Series instrument (Malvern Instruments, Malvern, UK). PTX concentrations were measured by highperformance liquid chromatography with ultraviolet detection at $227 \mathrm{~nm}$ on an Agilent 1100 series instrument (Agilent Technologies). The following parameters were used: mobile phase (acetonitrile to water, 70:30), column (CC 125/4 Nucleod UR 100-5 C18) and standard solutions for calibration $(5-100 \mu \mathrm{g} / \mathrm{mL}$ PTX diluted in acetonitrile; correlation coefficient $=0.99$, limit of detection $=1.6 \mu \mathrm{g} / \mathrm{mL}$, limit of quantification $=5 \mu \mathrm{g} / \mathrm{mL}$, coefficients of variation $<4.5 \%$ ). PTX was extracted from the NPs as previously described. ${ }^{9}$ Briefly, NPs were dissolved in acetonitrile (50:50), vortexed for $5 \mathrm{~min}$ and centrifuged at $4,000 \mathrm{rpm}$ for $10 \mathrm{~min}$. The supernatant was collected and used to measure the drug content. Encapsulation efficiency of PTX was calculated as the ratio of the encapsulated drug compared with the initial amount of the drug.

\section{In vitro studies}

\section{Cell cultures}

U87MG cells were cultured in EMEM supplemented with $10 \% \mathrm{FBS}, 100 \mathrm{U} / \mathrm{mL}$ penicillin $\mathrm{G}$ sodium and $100 \mu \mathrm{g} / \mathrm{mL}$ streptomycin sulfate at $37^{\circ} \mathrm{C}$ in a $5 \% \mathrm{CO}_{2} / 95 \%$ air-humidified incubator.

\section{Cellular uptake}

A cellular uptake study was performed using FITC-labeled PLGA-NPs. U87MG cells were seeded at a density of $10^{5}$ cells $/ \mathrm{mL}$ in a six-well plate with coverslips previously coated with poly-D-lysine $(0.1 \mathrm{mg} / \mathrm{mL})$. After $24 \mathrm{~h}$ of incubation, the culture medium was replaced with culture medium containing PTX/SPIO-NPs (1-10 $\mu \mathrm{g} / \mathrm{mL}$ PTX concentration) and was incubated for $1 \mathrm{~h}$. Supernatant was aspirated from the wells, and the cells were fixed with $4 \%$ formaldehyde for $20 \mathrm{~min}$. The coverslips were then placed onto slides with mounting solution containing DAPI. ${ }^{20}$ The cellular uptake of the NPs was determined using a fluorescence Axioskop 40 microscope fitted with two excitation filters, blue $(340 / 380 \mathrm{~nm})$ and green $(470 / 490 \mathrm{~nm})$, and images were obtained with an Axiocam MRc5 camera (Carl Zeiss Meditec AG, Jena, Germany).

\section{Evaluation of intracellular iron oxide internalization by Prussian blue staining}

Internalized intracellular iron oxide can be visualized by Prussian blue staining. U87MG cells were seeded and incubated as previously described for cellular uptake studies. ${ }^{13}$ After $24 \mathrm{~h}$ of incubation, the culture medium containing SPIO-NPs ( $80 \mu \mathrm{g} / \mathrm{mL}$ Fe concentration) was added and incubated for $2 \mathrm{~h}$. Cells were washed with PBS and fixed with $4 \%$ formaldehyde for $15 \mathrm{~min}$. Cells were washed again and incubated for 30 min with Prussian blue working solution with an equal volume of $2 \% \mathrm{HCl}$ and $2 \%$ potassium ferrocyanide(II) trihydrate, followed by washing with PBS and water. Intracellular iron oxide internalization was evaluated using an Axioskop 40 microscope, and images were acquired with an Axiocam MRc5 camera.

\section{In vivo studies}

All experiments were conducted according to the Belgian national regulation guidelines, with approval from the animal 
care ethical committee of the Université catholique de Louvain (2014/UCL/MD/004).

\section{Orthotopic U87MG tumor model}

The U87MG orthotopic tumor model was established by following a previously reported protocol ${ }^{21,22}$ with a few modifications. Naval Medical Research Institute (NMRI) nude mice (female, 6 weeks old) were obtained and acclimatized following standardized animal experimentation protocols. Mice were anesthetized with an intraperitoneal injection of xylazine $(13 \mathrm{mg} / \mathrm{kg})$ and ketamine $(100 \mathrm{mg} / \mathrm{kg})$. On day 0 , after positioning into a stereotaxic frame, mice received a $3 \mu \mathrm{L}$ intracranial injection of U87MG cells in the culture medium $\left(3 \times 10^{4}\right.$ cells $)$ into the right frontal lobe (striatum; coordinates: $2.1 \mathrm{~mm}$ lateral from bregma, $0.5 \mathrm{~mm}$ anterior and $3 \mathrm{~mm}$ deep from the outer border of the cranium) using a $5 \mu \mathrm{L} \mathrm{Hamilton}^{\circledR}$ syringe (Sigma-Aldrich Co.) fitted with a $26 \mathrm{G}$ needle.

\section{Magnetic resonance imaging}

The tumor presence, location and volume were determined by MRI using an 11.7 T Biospec small animal MR scanner (Bruker Optik GmbH). Mice were anesthetized with 2.5\% isoflurane (for induction) and were maintained with 1\% while scanning. Respiration was monitored throughout the experiment, and animal body temperature was maintained by a hot water circulation system. $T_{2}$-weighted MRI scans (rapid acquisition with refocused echo [RARE] sequence, repetition time $[\mathrm{TR}]=2,500 \mathrm{~ms}$, effective echo time $\left[\mathrm{TE}_{\text {eff }}\right]=30 \mathrm{~ms}$, RARE factor $=8$, matrix size $=256 \times 256$, field of view $[\mathrm{FOV}]=2 \times 2 \mathrm{~cm}$, number of continuous slices $=25$, slice thickness $=0.3 \mathrm{~mm}$, in-plane resolution $=78 \mathrm{~m}^{2}$ ) were used to monitor tumor growth. The tumor volume was determined by the sum of the cross-sectional disks of a manually drawn region of interest (ROI) on all slices in the axial orientation using Paravision 6.1 software (Bruker Optik GmbH). This protocol was used to follow the tumor development, and localization in the right striatum was determined with MRI prior to treatment. ${ }^{23}$

\section{Evaluation of BBB disruption}

$\mathrm{BBB}$ disruption was evaluated by using the $\mathrm{T}_{1}$ contrast agent Gd-DOTA in U87MG tumor-bearing mice with tumor volumes of $\sim 0.2 \mathrm{~mm}^{3}$. Gd-DOTA was injected intravenously at a dose of $16.4 \mathrm{mg} / \mathrm{kg}$ body weight. $\mathrm{T}_{1}$-weighted RARE sequence MRI scans with TR of $800 \mathrm{~ms}$ and TE $\mathrm{eff}_{\text {f }}$ of $5.5 \mathrm{~ms}$ were used to obtain pre- and postcontrast images. BBB disruption was evaluated by quantification of the differences in the signal intensities on ROIs of tumor tissue and normal tissue of the pre- and postcontrast images in each subject. ${ }^{23,24}$ The normal values were measured from contralateral side of tumor.

\section{Ex vivo biodistribution studies}

The fate of the biodistribution of NPs was evaluated by the quantification of SPIO for different conditions:

1. Healthy mice versus GBM-bearing mice. NMRI mice and GBM-bearing nude mice (GBM tumor volume $0.2 \mathrm{~mm}^{3}$; $\mathrm{n}=5$ and $\mathrm{n}=7$, respectively) were intravenously injected with PTX/SPIO-NPs at an equivalent PTX concentration (5 mg/kg).

2. GBM-bearing mice with PS80-coated NPs.

3. GBM-bearing mice with and without magnetic targeting. U87MG tumor-bearing NMRI nude mice $(n=7)$ were intravenously injected with PTX/SPIO-NPs at an equivalent PTX concentration $(5 \mathrm{mg} / \mathrm{kg}$ ) with and without applying an external magnetic field.

For the application of the magnetic field, disk-shaped ( $1.7 \mathrm{~g}$ ) neodymium magnets (Supermagnete, Gottmadingen, Germany) with a remanence $\left(\mathrm{B}_{\mathrm{r}}\right)$ of $1.4 \mathrm{~T}$ were placed on the heads of mice and held in place with the aid of cotton gage, adhesive tape and mouse Elizabethan collars (Harvard apparatus, Les Ulis, France; Figure S1).

All the mice were sacrificed $24 \mathrm{~h}$ after injection to collect brain, liver, spleen and lung samples. All tissue samples were snap-frozen before lyophilization. The lyophilized samples were ground into fine powder, and SPIO was quantified by ESR spectroscopy. ${ }^{14}$

\section{In vivo toxicity evaluation and histology}

Swiss mice (female, 6 weeks old) were randomly divided into three groups $(n=6)$. Each group received one intravenous injection of PTX/SPIO-NPs ( $5 \mathrm{mg} / \mathrm{kg}$ PTX), Taxol ${ }^{\circledR}$ (5 mg/kg PTX) (Auribindo pharma ltd., Hyderabad, India) or saline. Body weight was monitored daily. Blood samples were collected after $24 \mathrm{~h}$ for hematologic and histochemical analysis. Aspartate aminotransferase (AST), alanine aminotransferase (ALT), blood urea nitrogen (BUN) and creatine kinase isoenzyme (CKMB) levels were assayed ${ }^{25}$ using a Fujifilm DRI-CHEM NX500i Analyzer (Fujifilm, Tokyo, Japan).

\section{In vivo antitumor efficacy}

The effect of PTX/SPIO-NPs on tumor growth was assessed by median survival rate in the orthotopic U87MG model. Tumor cell inoculation was performed as aforementioned. Seven days after tumor implantation (tumor volume $\sim 0.2 \mathrm{~mm}^{3}$ ), 


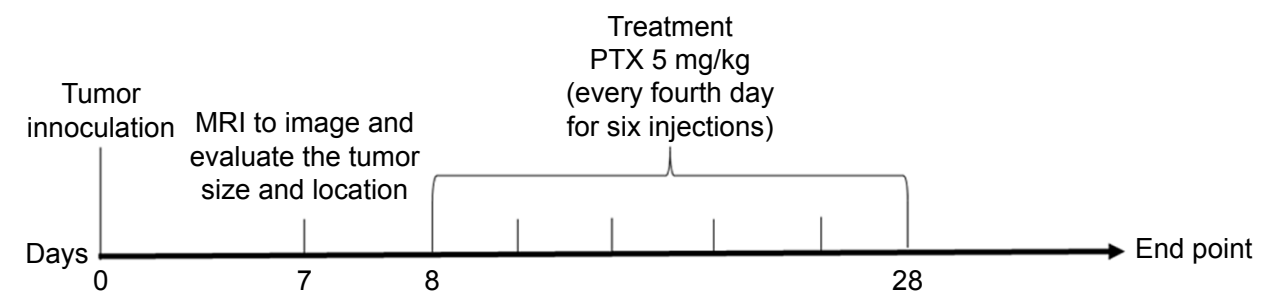

Figure I Protocol to study the anti-tumor efficacy of PTX/SPIO-NPs with or without magnetic targeting.

Note: Anti-tumor efficacy study timeline.

Abbreviations: MRI, magnetic resonance imaging; NPs, nanoparticles; PLGA, poly(lactic-co-glycolic acid); PTX, paclitaxel; SPIO, super paramagnetic iron oxide.

tumor location and volume were assessed by MRI, after which mice were randomly assigned into groups. Figure 1 shows the timeline of the experimental plan. Three groups were defined (seven mice per group): group 1, injection of PBS; group 2, injection of PTX/SPIO-NPs at a dose of $5 \mathrm{mg} /$ $\mathrm{kg} \mathrm{PTX}{ }^{26}$ and $14 \mathrm{mg} / \mathrm{kg} \mathrm{Fe}$ (passive targeting); group 3, the same injection of PTX/SPIO-NPs with magnetic targeting with neodymium magnets with a remanence of $1.4 \mathrm{~T}$ during $4 \mathrm{~h}$. Treatments were injected through the tail vein and repeated every fourth day for a total of six injections. The end point of the experiment was determined as $20 \%$ weight loss or $10 \%$ weight loss with tumor-related sickness.

\section{Statistical analyses}

All results are expressed as mean $\pm \mathrm{SD}$. Two-way analysis of variance with Bonferroni posttests and Kaplan-Meier survival rate curves were performed using the software Graph Pad Prism 5 for Windows (GraphPad Software Inc., La Jolla, CA, USA) to demonstrate significant differences among treatment groups.

\section{Results and discussion Physicochemical properties}

PTX and SPIO-loaded-PLGA-based NPs were prepared using an emulsion-diffusion-evaporation method with PVA as a stabilizer. Table 1 presents the physicochemical characterization (size, PDI, zeta potential, encapsulation efficiency, drug loading and iron loading) of the prepared NPs. Prepared SPIO and SPIO encapsulated in NPs showed superparamagnetic properties that are advantageous for magnetic targeting. Figure 2 shows the attraction of SPIO to the neodymium magnet. The Fe concentration of the SPIO was found to be $7.7 \mathrm{mg} / \mathrm{mL}$, and this value is in agreement when quantified by ESR or ICP-MS. Nuclear magnetic relaxation dispersion profiles, transmission electron microscopic images and relaxometry studies highlighting the superparamagnetic properties of prepared SPIO were previously reported by our group. ${ }^{13}$ The average size of the PTX/SPIO-NPs was $250 \pm$ $20 \mathrm{~nm}$, which is consistent with our previous reports. ${ }^{13,14}$ The prepared NPs were in the appropriate size range to accumulate in tumor tissues, as it has been established that the optimal particle size for the EPR effect ranges from 20 to $400 \mathrm{~nm} .{ }^{27}$ The PDI showed a narrow size distribution of $<0.2$. The zeta potential of the PTX/SPIO-NPs was $-18 \pm$ $5 \mathrm{mV}$. Coating with PS80 increased the negative surface potential to $-34 \pm 3 \mathrm{mV}$, suggesting the adsorption of PS80 on the surface of the PTX/SPIO-NPs.

Encapsulation efficiencies of PTX were calculated as $31 \% \pm 2 \%$ and $30 \% \pm 6 \%$ for the PTX-NPs and PTX/SPIO-NPs, respectively. PTX loading was found to be $\sim 0.8 \mathrm{mg} / 100 \mathrm{mg}$ polymer in both the PTX-NPs and PTX/SPIO-NPs groups. Drug loading was not affected by the coencapsulation

Table I Physicochemical characterization of NPs $(n \geq 3)$

\begin{tabular}{|c|c|c|c|c|}
\hline & PLGA-NPs & SPIO-NPs & PTX-NPs & PTX/SPIO-NPs \\
\hline Size $(\mathrm{nm})$ & $215 \pm 4$ & $225 \pm 12$ & $203 \pm 4$ & $250 \pm 20$ \\
\hline PDI & 0.07 & 0.09 & 0.07 & 0.11 \\
\hline Zeta potential (mV) & $-8.6 \pm 1$ & $-13 \pm 5$ & $-21 \pm 3$ & $-18 \pm 5$ \\
\hline Drug encapsulation efficiency (\%) & NA & NA & $31 \pm 2$ & $30 \pm 6$ \\
\hline Drug loading (\%) & NA & NA & $0.85 \pm 0.3$ & $0.87 \pm 0.2$ \\
\hline Iron loading (\%) & NA & $4 \pm 1$ & NA & $3 \pm 1$ \\
\hline
\end{tabular}

Notes: physicochemical characterization of PLGA based PEGylated NPs (PLGA-NPs) loaded with either SPIO (SPIO-NPs), PTX (PTX-NPs), PTX and SPIO (PTX/ SPIO-NPs).

Abbreviations: NA, not applicable; NPs, nanoparticles; PDI, polydispersity index; PLGA, poly(lactic-co-glycolic acid); PS80, polysorbate 80; PTX, paclitaxel; SPIO, super paramagnetic iron oxide. 
A
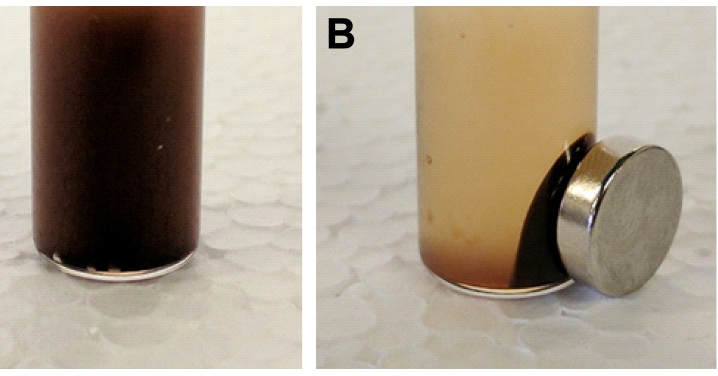

Figure 2 SPIO in the absence (A) and presence (B) of a commercial neodymium magnet $\left(B_{r}=1.4 \mathrm{~T}\right)$.

Note: Separation of SPIO from the dispersion occurred in $<15 \mathrm{~s}$.

Abbreviation: SPIO, super paramagnetic iron oxide.

of SPIO. Although the emulsion technique can result in high drug-loading values, the overall process is affected by several in-process parameters, such as duration of sonication and centrifugation, possible interactions between polymer/stabilizer and between the drug/SPIO, surface coating, filtration and washing steps that could influence the encapsulation efficiency and polymer loss. ${ }^{28}$ Some of the aforementioned factors might have contributed to the $30 \%$ encapsulation efficiency in our formulation. Nevertheless, this formulation allows for the delivery of a therapeutic dose of PTX (5 mg/kg).

\section{In vitro cellular studies}

To assess the uptake of the PTX/SPIO-NPs in U87MG cells, NPs were prepared with a covalently grafted FITC
PLGA (green fluorescence), and to visualize cell nuclei, DAPI staining (blue fluorescence) was used. Figure 3A shows the dose-dependent internalization of NPs. With increasing amounts of NPs (from 1 to $10 \mu \mathrm{g} / \mathrm{mL}$ PTX), FITC increased. FITC allowed the visualization of the internalized polymeric NPs by cells, suggesting a broad distribution of NPs within the cytosol (Figure 3B). The same pattern of dose-dependent cellular uptake has been reported in the literature. $^{29}$

SPIO (Fe) staining also allowed the visualization of NPs within the cells. Figure 3D shows the entrapment of SPIO (intense blue color) in cells incubated with PTX/SPIO-NPs $(80 \mu \mathrm{g} / \mathrm{mL} \mathrm{Fe})$, while no blue color was observed in cells of the control group (without NPs; Figure 3C), consistent with previous reports..$^{30}$ Thus, both experiments confirmed that the NPs are internalized within the U87MG cells.

We have also evaluated in vitro cytotoxicity of NPs on U87MG cell line. The results showed the concentrationdependent cytotoxic effect of PTX. Furthermore, inclusion of controls (unloaded PLGA-NPs, PTX-NPs and SPIO-NPs) confirmed that the cytotoxic effect was solely due to PTX. The $\mathrm{IC}_{50}$ value of PTX/SPIO-NPs after $48 \mathrm{~h}$ was found to be $1 \mathrm{ng} / \mathrm{mL}$ (data not shown).

To exert therapeutic effects, PTX/SPIO-NPs must be internalized and sustainably retained within the tumor cells, as the mode of action of PTX is interference of the mitotic spindle assembly. ${ }^{31}$ There are several mechanisms of cellular
A

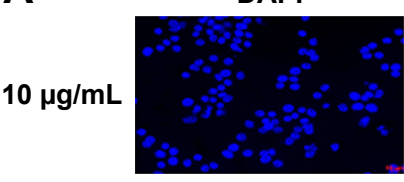

$5 \mu \mathrm{g} / \mathrm{mL}$
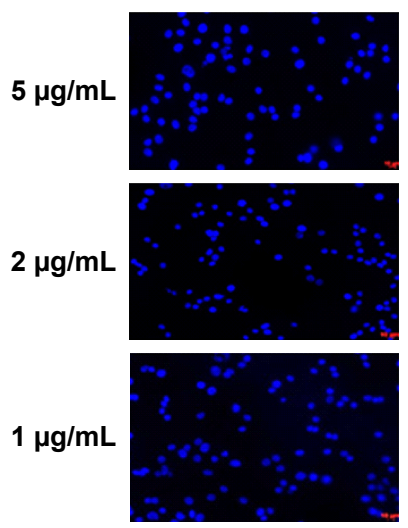

FITC
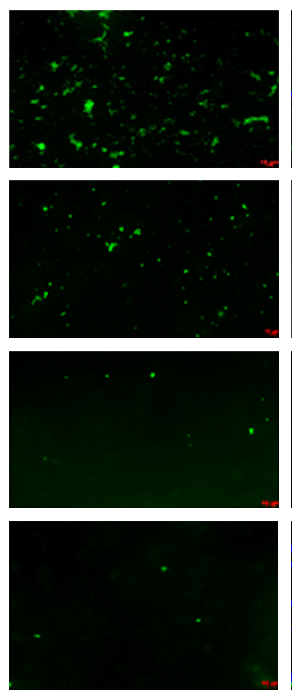

DAPI + FITC
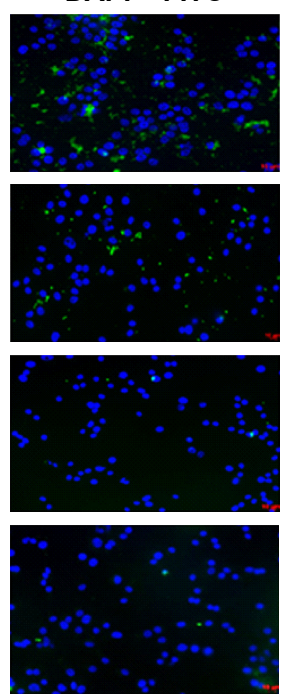

B

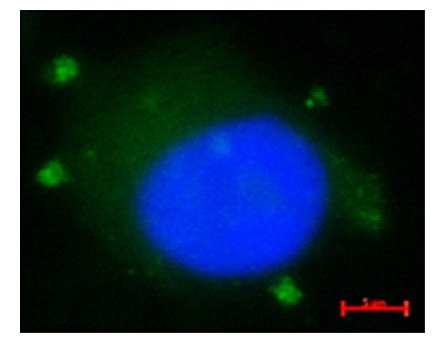

C

D

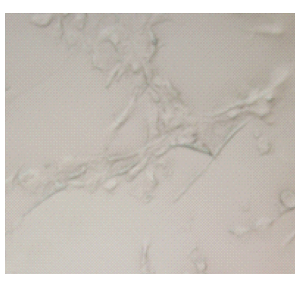

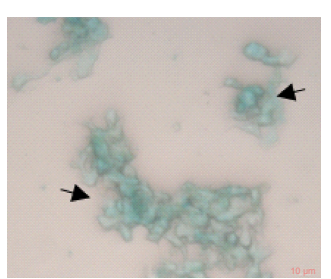

Figure 3 In vitro cellular uptake studies $(n=3)$.

Notes: (A) Fluorescent images of FITC-labeled PLGA-NPs at different concentrations of PTX $(\mathrm{I}-10 \mu \mathrm{g} / \mathrm{mL})$ after I h of incubation. (B) FITC green fluorescence around the blue color of DAPI-stained nuclei clearly exhibited the uptake of FITC-labeled PLGA-NPs. (C) Prussian blue staining of untreated cells (control). (D) Prussian blue staining of internalized SPIO-NPs $(80 \mu \mathrm{g} / \mathrm{mL}$ Fe). The blue color of cells is due to a chemical reaction occurring between internalized iron and Prussian blue stain. PLGA-NPs, PLGAbased NPs; SPIO-NPs, SPIO-loaded NPs.

Abbreviations: FITC, fluorescein isothiocyanate; NPs, nanoparticles; PLGA, poly(lactic-co-glycolic acid); PTX, paclitaxel; SPIO, super paramagnetic iron oxide. 
internalization of NPs. The physicochemical properties of NPs (size, shape and surface chemistry) influence the intensity and path of cellular internalization. ${ }^{32}$ In the case of PTX/ SPIO-NPs, there are no ligands attached to the NP surface, and hence specific receptor-mediated uptake may not occur. Fluid-phase pinocytosis or clathrin-mediated nonspecific receptor-mediated endocytosis mechanisms may facilitate the internalization of PTX/SPIO-NPs. ${ }^{33,34}$ The size $(250 \pm 20 \mathrm{~nm})$ and zeta potential $(-18 \pm 5 \mathrm{mV})$ of the NPs are within the allowed limits that enable cellular internalization by these mechanisms. ${ }^{32,35}$ In vitro testing may not entirely replicate in vivo biological processes. However, in vitro results can provide some preliminary evidence to present the advantages of nanodrug formulations over the free drug. Further studies using several specific uptake inhibitors can concretely confirm the uptake mechanism of PTX/SPIO-NPs.

\section{Evaluation of BBB disruption in a GBM orthotopic tumor model}

An ideal GBM model should closely resemble human GBM in its morphology, invasiveness, angiogenesis and BBB disruption to allow clinically relevant studies of tumor behavior and therapeutic drug efficiency. ${ }^{36}$ The loss of BBB integrity is very important for assessing novel GBM treatment approaches/drug delivery systems upon systemic administration. Reports in the literature also state that tumor lesions larger than $0.5 \mathrm{~mm}$ or $0.2 \mathrm{~mm}^{2}$ show BBB permeability as a consequence of disrupted BBB. ${ }^{37,38}$ The U87MG tumor model is known to present moderate invasiveness coupled with tumor-induced necrosis and vascular alterations, which makes this a suitable GBM model for therapeutic agent studies. ${ }^{39,40}$ Contributing to these tumor model features, we have tested the $\mathrm{BBB}$ disruption in the U87MG tumor model $(n=4)$. To be consistent, in all our investigations, the tumor volume was $\sim 0.2 \mathrm{~mm}^{3}$.
$\mathrm{T}_{1}$-weighted MRI after injection of Gd-DOTA contrast agent was performed on GBM-bearing mice presenting a GBM volume of $0.2 \mathrm{~mm}^{3}$. The enhancement of signal intensity in the tumor region (bright area of the tumor in pre- and postcontrast images is marked by circles) in a representative postcontrast image can be clearly visualized the enhancement of signal intensity in the tumor region (highlighted area of the tumor in pre- and postcontrast images) in a representative postcontrast image can be clearly visualized (Figure 4A). This is due to the accumulation of Gd-DOTA paramagnetic chelates in the tumor region, which indicate a loss of BBB integrity, while in normal conditions, Gd-DOTA cannot cross the BBB. The enhanced signal intensity can also be quantified on ROIs of tumor tissue and normal tissue within the pre- and postcontrast images, and the result showed statistical significance in the tumor tissue compared with the normal tissue $(p<0.001$; Figure 4B). There is no enhancement of signal intensity in the normal tissue. The high variability of signal intensity values in each individual subject in the test groups inhibited the use of collective statistics. However, a significant difference in signal intensity values between the tumor tissue and normal tissue could be observed for each individual animal in the group. With this result, we showed that the BBB was disrupted when the GBM presented a volume of $\sim 0.2 \mathrm{~mm}^{3}$, and this validated U87MG model was used for the following in vivo studies.

\section{Ex vivo biodistribution studies}

Biodistribution studies provide essential information about the accumulation of therapeutic or diagnostic agents in different vital organs and tumors. ${ }^{41}$ In the current study, different strategies such as surface modifications and the application of an external magnetic field have been tested to enhance the accumulation of NPs by crossing the disrupted
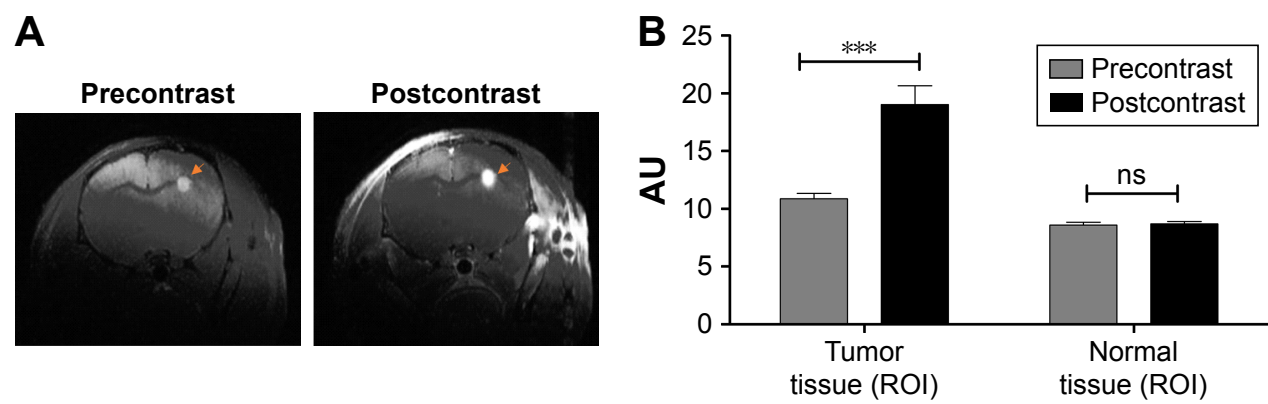

Figure 4 In vivo BBB disruption evaluation $(n=4)$.

Notes: (A) Pre- and postcontrast MRI images of brain tissue highlighting the tumor region (right). Accumulation of T, contrast agent (Gd-DOTA I6.4 mg/kg) enhanced the signal intensity can be visualized from pre- and postcontrast images. (B) Quantification of signal intensity in ROI of tumor tissue versus normal tissue. A significant difference in signal intensity values was observed between pre- and postcontrast tumor tissue, whereas no difference in the normal regions could be observed for each individual animal in the group. $* * * p<0.001$.

Abbreviations: BBB, blood-brain barrier; Gd-DOTA, gadoterate meglumine (Dotarem); MRI, magnetic resonance imaging; ns, no significance; ROI, region of interest. 
BBB in GBM-bearing mice. One of the strategies explored was surface modification of the NPs with surfactants such as PS80. A few reports have demonstrated that drug-loaded polymeric NPs coated with PS80 can cross the BBB and be taken up by brain endothelial cells. ${ }^{42,43}$ More interestingly, magnetic NPs can be guided to the tumor site using an externally applied magnetic field to further enhance drug delivery at a targeted site. NP concentrations in the excised tissue samples of brain and vital organs were quantified using ESR spectroscopy.

Biodistribution studies of PTX/SPIO-NPs in healthy mice served as a control for comparitive analysis with GBM-bearing mice, and the quantified amounts of Fe in brain tissue are shown in Figure 5A. The brain accumulation of PTX/SPIO-NPs was twofold higher in GBM-bearing mice compared with healthy mice ( $p<0.05$; Figure 5A). Enhancement of Fe in brain tissue of GBM-bearing mice can be attributed to the presence of a leaky BBB in GBMbearing mice. It has been reported that GBM induces a series of events mediated by several glioma-derived factors such as TGF- $\beta 2$, caveolin-1, reactive oxygen species, aquaporins (especially Aqp-4) and proinflammatory peptides. These glioma-derived factors eventually lead to the degradation of tight junctions and downregulation of endothelial tight junction proteins, resulting in glioma-induced impairment of the BBB. ${ }^{44}$ In addition to the loss of BBB integrity, the physicochemical properties of the NPs could have contributed to the accumulation of the NPs.

PTX/SPIO-NPs and PS80-coated PTX/SPIO-NPs (PTX/ SPIO-PS80-NPs) were injected into GBM-bearing mice. Both PTX/SPIO-NPs and PTX/SPIO-PS80-NPs showed greater accumulation in the brain tissues of GBM-bearing mice than in the control mice. There was no significant difference between the accumulation of coated and uncoated NPs in the brain tissues.

To take advantage of the magnetic properties of SPIO in the NPs and enhance the accumulation of NPs in brain tissue by magnetic targeting, PTX/SPIO-NPs were injected in GBM-bearing mice, and an external magnetic field was applied for $4 \mathrm{~h}$. The magnetic targeting of NPs showed a threefold increase in the accumulation of NPs in the brain compared with no magnetic field in the control group, thanks to the superparamagnetic properties of SPIO (Figure 5A). In the literature, a variable increase in the accumulation of NPs with magnetic targeting has been described. Chertok et $\mathrm{al}^{45}$ reported an 11.5-fold increase in the accumulation of NPs in tumor tissue compared to that of controls after $50 \mathrm{~min}$ of treatment administration. Furthermore, the success of magnetic targeting is limited by inadequate magnetic gradients, magnet position and time, as the magnetic targeting would be more effective in regions closer to the magnets.
A

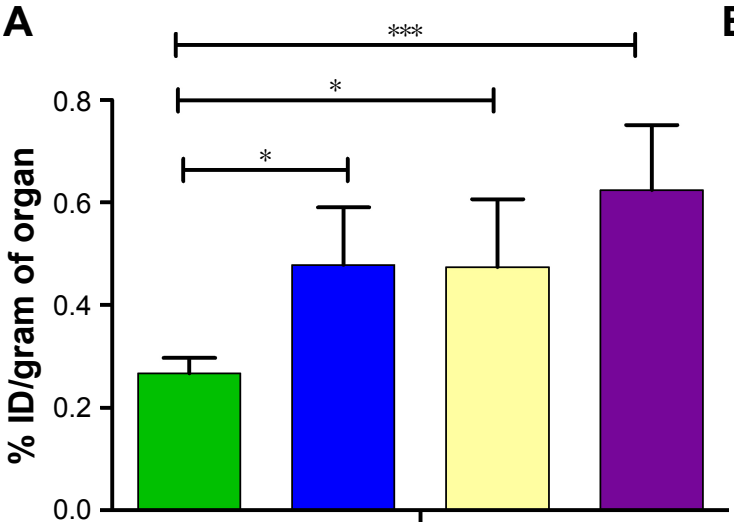

B

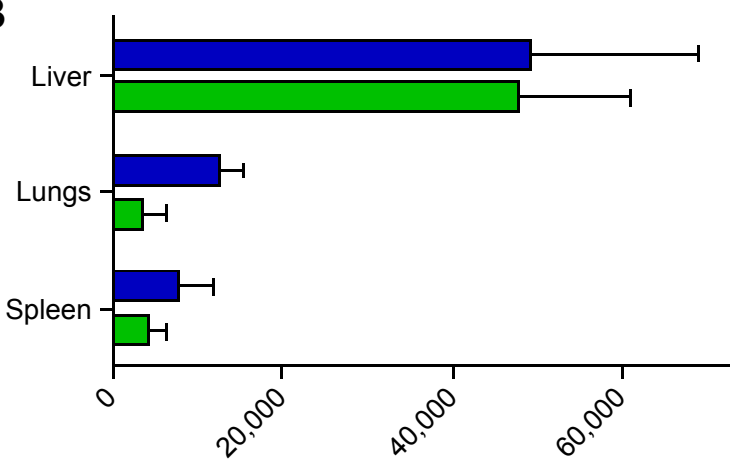

$\mathrm{Fe}$ (ng)/organ

Healthy mice/PTX/SPIO-NPs $\square$ U87MG tumor mice/PTX/SPIO-NPs U87MG tumor mice/PTX/SPIO-PS80-NPs

U87MG tumor mice/PTX/SPIO-NP/magnetic targeting

Figure 5 The ex vivo biodistribution study $(n=7)$ at $24 \mathrm{~h}$ after the injection of PTX/SPIO-NPs was performed by quantification of Fe (SPIO) using ESR spectroscopy in brain tissue from healthy and GBM-bearing mice.

Notes: (A) Ex vivo biodistribution of PTX/SPIO-NPs or PTX/SPIO-PS80-NPs in the brain of healthy or GBM-bearing mice ( $\mathrm{n}=7$ ) with or without the application of an external magnetic field. With the disrupted BBB in the GBM-bearing mice, the accumulation of NPs was twofold higher than that in the control group, and magnetic targeting resulted in the highest iron ( $\mathrm{Fe}$ ) enhancement among all the groups. (B) Ex vivo biodistribution in the liver, spleen and lungs (RES organs). There were no significant differences in uptake among different organs for healthy mice or GBM-bearing mice. PTX/SPIO-PS80-NPs, PS80-coated PTX/SPIO-NPs; PTX/SPIO-NPs, PTX/SPIO loaded PLGA-based NPs. *p $<0.05$, *** $p<0.001$.

Abbreviations: BBB, blood-brain barrier; ESR, electron spin resonance; GBM, glioblastoma; ID, injected dose; NPs, nanoparticles; PLGA, poly(lactic-co-glycolic acid); PS80, polysorbate 80; PTX, paclitaxel; RES, reticuloendothelial system; SPIO, super paramagnetic iron oxide. 
Further investigations must be performed to improve magnetic targeting applications.

The accumulation of $\mathrm{Fe}$ in the spleen, liver and lungs showed varied quantities (higher uptake in the liver) with no significant differences in uptake among the different organs in healthy mice or GBM-bearing mice (Figure 5B). In addition, our group previously reported that blank NPs (without PTX) have shown similar distribution profiles to those of the PTX/SPIO-NPs. ${ }^{14}$ NPs with a hydrodynamic diameter smaller than $10 \mathrm{~nm}$ are prone to tissue extravasation and are primarily cleared in the renal pathway, whereas NPs with larger sizes $(>10 \mathrm{~nm})$ undergo opsonization and are cleared from systemic circulation via the reticuloendothelial system/ hepatobiliary pathway. ${ }^{46}$ However, the rate of clearance is decreased by PEGylation due to steric resistance of opsonization and macrophage uptake processes, thereby greatly enhancing the resistance toward RES interactions. ${ }^{47}$ Thus, the physicochemical properties of the NPs alter the circulation half-lives of NPs to have greater exposure to target tissues.

The question arising would be about iron overload in the liver with SPIO in the formulation. So far, there have been no reports establishing SPIO toxicity. However, there have been reports showing that SPIO is retained in the liver for several weeks, and that excess SPIO in the liver does not impair liver function or promote a significant immunotoxicity response. ${ }^{8,48,49}$ Thus, PTX/SPIO-NPs necessitate a toxicity evaluation to demonstrate in vivo safety.

\section{In vivo safety evaluation}

Evaluation of the toxicological effects of nanomaterial-based diagnostics and therapeutics is of critical importance and is still a long way from being fully understood..$^{50}$ PLGA-based
NPs are well known for their biocompatibility, but it is necessary to ensure that the developed PTX/SPIO-loaded PLGA nanocarriers do not exhibit major toxicological problems, in particular hepatotoxicity due to high accumulation in the liver. Systemic toxicity of PTX/SPIO-NPs at a single dose of $5 \mathrm{mg} / \mathrm{kg}$ PTX was evaluated in healthy mice. Compared to the control (saline) group, no death and no obvious body weight loss were observed in all tested groups during the safety evaluation study period. Moreover, most of the intravenously injected NPs are taken up and taken up and eliminated by RES organs which includes liver and kidneys. ${ }^{25}$ Therefore, AST and ALT for hepatic function, BUN for kidney function and CKMB as cardiac markers were measured on day 1 and day 7 after treatment injection.

On day 1 (Figure 6A), AST levels of the NP group were higher $(239 \pm 157 \mathrm{U} / \mathrm{L} ; p<0.05)$ than the control groups with saline $(55 \pm 10 \mathrm{U} / \mathrm{L})$ and Taxol $(74 \pm 28 \mathrm{U} / \mathrm{L})$. The transient increase in AST levels in serum upon the administration of PTX/SPIO-NPs appeared to be a general short-term response due to the hepatic accumulation of the injected NPs. These measured AST levels are significantly within the normal limits (39-262 U/L) $)^{51}$ and show that liver function is not significantly altered. Moreover, histopathological evaluation of liver tissue of the PTX/SPIO-NP group showed no or mild necrosis and no inflammation and accumulated SPIO in Kupffer cells after $24 \mathrm{~h}$, whereas that of Taxol (reference) group showed moderate necrosis with no inflammation (data not shown). For CKMB, higher levels were detected in the NP and Taxol groups than the saline group, but there was no statistically significant difference between groups $(p>0.05)$. The amounts of ALT and BUN in all the groups remained low and did not present any significant differences compared with the saline group $(p>0.05)$.
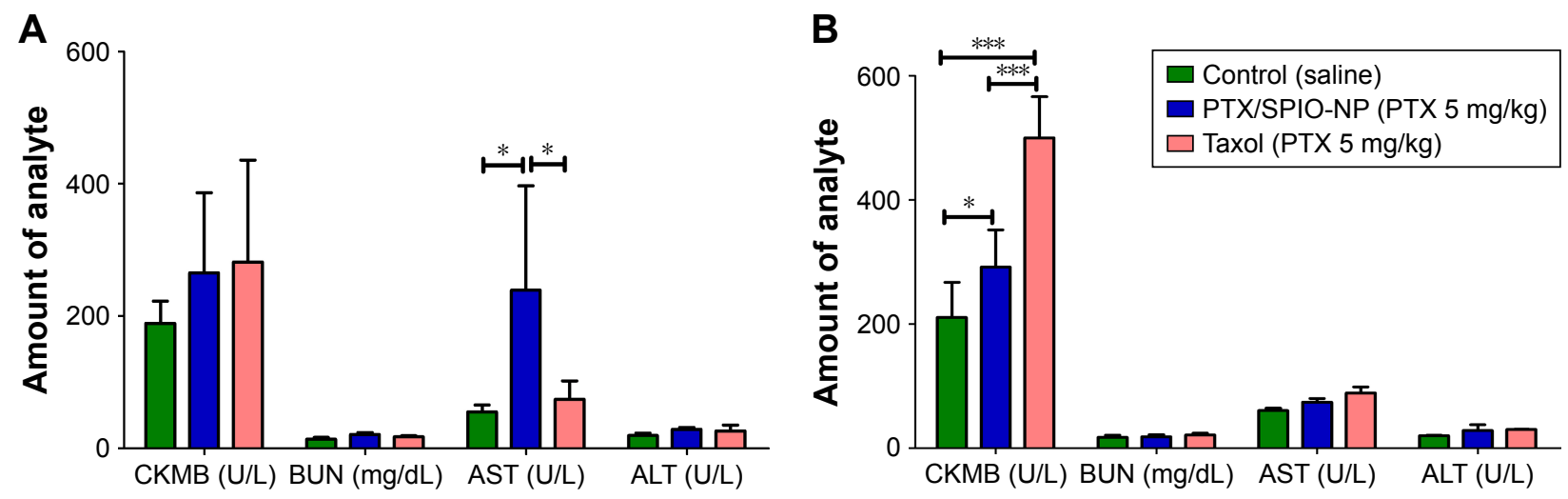

Figure 6 In vivo safety evaluation of PTX/SPIO-NPs in healthy mice $(n=6)$.

Notes: (A) Evaluation of biochemical parameters (AST, ALT, BUN and CKMB) I day after treatment injection. (B) Evaluation of biochemical parameters (AST, ALT, BUN and CKMB) 7 days after treatment injection. PTX/SPIO-NPs, PTX/SPIO loaded PLGA-based NPs. ${ }^{*} p<0.05$, *** $p<0.001$.

Abbreviations: ALT, alanine aminotransferase; AST, aspartate aminotransferase; BUN, blood urea nitrogen; CKMB, creatine kinase isoenzyme; NPs, nanoparticles; PLGA, poly(lactic-co-glycolic acid); PTX, paclitaxel; SPIO, super paramagnetic iron oxide. 
At day 7 (Figure 6B), there was no increase in the levels of the three parameters such as BUN, AST or ALT compared to the saline group. Interestingly, after 1 week, the AST levels returned to normal, indicating that the PTX/SPIO-NPs did not induce hepatic toxicity. The highest CKMB levels were observed in the group injected with Taxol $(p<0.001)$. Although the mice treated with PTX/SPIO-NPs showed higher CKMB levels than the saline group ( $p<0.05)$, these CKMB values are much lower when compared to positive control (Taxol). In addition, histopathological evaluation showed no alterations in liver tissues of both groups (data not shown). Thus, the results indicate that NPs did not exert toxicity higher than the positive control treatment (Taxol). Although major amounts of the accumulation of NPs in the liver were shown in the ex vivo biodistribution study, our in vivo safety evaluation results have shown no significant toxicity and are in compliance with literature reports. ${ }^{51}$ Nevertheless, further long-term studies must be performed to evaluate the NP-induced toxicity upon single- and multipledose administrations.

\section{In vivo antitumor efficacy}

In vivo antitumor efficacy of multifunctional NPs was performed as a blinded experiment evaluated in a U87MG orthotopic tumor model. Tumor progression was assessed by MRI before treatment (day 7) and during treatment (day 21; Figure 7A). Figure 7B shows the Kaplan-Meier survival curves $(n \geq 7)$ of the control, passive targeting and the magnetic targeting groups. The median survival time of mice treated with PTX/SPIO-NPs and with magnetic targeting was significantly prolonged (49 days) when compared to the mice that underwent passive targeting (41 days; $p<0.05$ ) and/or saline treatment (44 days; $p<0.05$ ). No significant difference was observed between the passive targeting group and the saline group. Although our model was evaluated for BBB disruption and the biodistribution data of passive targeting have shown statistically significant amounts of NPs traced in the brain tissue, we suppose that these amounts were not sufficient to limit the tumor growth or to prolong the survival time. This could be due to the highly heterogeneous and ambiguous EPR effect. Furthermore, tumors such as GBM are well known to show intratumor heterogeneity, that negatively effects the treatment outcome. Passive targeting is merely based on the EPR effect of drug distribution by blood circulation and extravasation via leaky vasculature of the tumor. ${ }^{52}$ Thus, passive targeting may result in subtherapeutic doses to tumor regions, lacking an advantage of prolonging survival periods or progression-free survival. Hence, the need for targeting strategies such as magnetic targeting is crucial.

Enhanced accumulation of NPs in targeted regions upon external magnetic field application implies that therapeutic/ significant amounts of PTX can reach the target site. Consequently, PTX/SPIO-NPs with magnetic targeting may prolong the survival time of GBM-bearing mice. Previously, many groups have demonstrated the in vivo efficacy of targeted and untargeted drug-loaded NPs. However, to the best of our knowledge, this study is the first to evaluate targeting strategies in terms of antitumor efficacy with biodegradable polymeric nanotheranostic particles in an orthotopic GBM model. This would allow a dual advantage for magnetic targeting and diagnostic properties of SPIO.
A

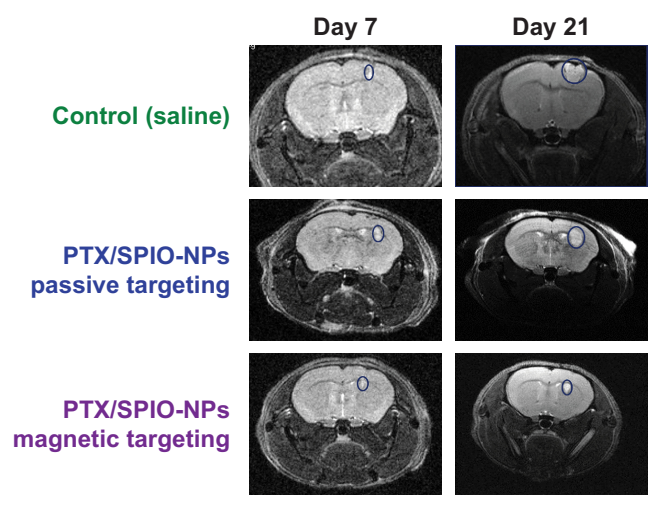

B

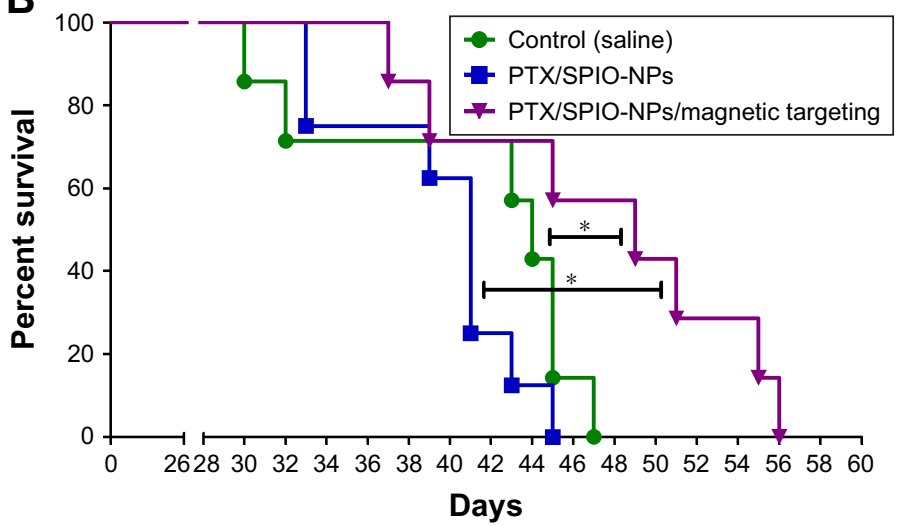

Figure 7 In vivo antitumor efficacy on survival.

Notes: (A) MRI images of the control, passive and magnetic groups before and during treatment (on day 7 and day 21 , respectively). (B) Kaplan-Meier survival curves ( $\mathrm{n} \geq 7$ ) of the control, passive and magnetic targeting groups. The median survival times were $44,4 \mathrm{I}$ and 49 days, respectively. The magnetic targeting group showed significant differences $(* p<0.05)$ when compared with both the control and passive groups. PTX/SPIO-NPs, PTX/SPIO loaded PLGA-based NPs.

Abbreviations: MRI, magnetic resonance imaging; NPs, nanoparticles; PLGA, poly(lactic-co-glycolic acid); PTX, paclitaxel; SPIO, super paramagnetic iron oxide. 


\section{Conclusion}

The aim of the study was to evaluate targeting strategies and their antitumor efficacies with PTX and SPIO-loaded PLGA-NPs. The cellular uptake of PTX/SPIO-NPs was concentration dependent. In an orthotopic GBM model, Gd-DOTA contrast agent confirmed that the BBB was disrupted, at least when tumor volume was $\sim 0.2 \mathrm{~mm}^{3}$. Magnetic targeting enhanced accumulation of PTX/ SPIO-NPs in the brain, as well as the antitumor efficacy, and prolonged the survival rate of mice compared with the passive targeting and saline treatments. Based on these results, it can be concluded that PTX/SPIO-NPs with magnetic targeting may be considered as an effective anticancer targeting strategy for GBM chemotherapy.

\section{Acknowledgments}

We thank Télévie, Belgian Fonds National de la Recherche Scientifique (FRS-FNRS) (7651916F), and Fondation contre le Cancer for funding this study. We express our earnest thanks to Kevin Vanvarenberg (Advanced Drug Delivery and Biomaterials, Université catholique de Louvain, Belgium) for his help with cellular studies, Sébastien Druart (Pôle de Néphrologie, Institut de Recherche Expérimentale et Clinique, Université catholique de Louvain, Belgium) for his help with the hematological and biochemical analyses and Dr Pierette Michel for histopathological evaluation.

\section{Disclosure}

The authors report no conflicts of interest in this work.

\section{References}

1. Gutkin A, Cohen ZR, Peer D. Harnessing nanomedicine for therapeutic intervention in glioblastoma. Expert Opin Drug Deliv. 2016;13(11): 1573-1582.

2. Hottinger AF, Stupp R, Homicsko K. Standards of care and novel approaches in the management of glioblastoma multiforme. Chin $J$ Cancer. 2014;33(1):32-39

3. Bernardi A, Braganhol E, Jäger E, et al. Indomethacin-loaded nanocapsules treatment reduces in vivo glioblastoma growth in a rat glioma model. Cancer Lett. 2009;281(1):53-63.

4. Sonali, Viswanadh MK, Singh RP, et al. Nanotheranostics: emerging strategies for early diagnosis and therapy of brain cancer. Nanotheranostics. 2018;2(1):70-86.

5. Stephen ZR, Kievit FM, Veiseh O, et al. Redox-responsive magnetic nanoparticle for targeted convection-enhanced delivery of $\mathrm{O}(6)$ benzylguanine to brain tumors. ACS Nano. 2014;8(10):10383-10395.

6. Tabatabaei SN, Girouard H, Carret A-S, Martel S. Remote control of the permeability of the blood-brain barrier by magnetic heating of nanoparticles: a proof of concept for brain drug delivery. J Control Release. 2015;206:49-57.

7. Fontes de Paula Aguiar M, Bustamante Mamani J, Klei Felix T, et al. Magnetic targeting with superparamagnetic iron oxide nanoparticles for in vivo glioma. Nanotechnol Rev. 2017;6(5):449-472.
8. Ma HL, Xu YF, Qi XR, Maitani Y, Nagai T. Superparamagnetic iron oxide nanoparticles stabilized by alginate: pharmacokinetics, tissue distribution, and applications in detecting liver cancers. Int J Pharm. 2008;354(1):217-226.

9. Danhier F, Lecouturier N, Vroman B, et al. Paclitaxel-loaded PEGylated PLGA-based nanoparticles: in vitro and in vivo evaluation. $J$ Control Release. 2009;133(1):11-17.

10. Zhao M, Liang C, Li A, et al. Magnetic paclitaxel nanoparticles inhibit glioma growth and improve the survival of rats bearing glioma xenografts. Anticancer Res. 2010;30(6):2217-2223.

11. Shah N, Chaudhari K, Dantuluri P, Murthy RSR, Das S. Paclitaxelloaded PLGA nanoparticles surface modified with transferrin and Pluronic ${ }^{\mathbb{P}} \mathrm{P} 85$, an in vitro cell line and in vivo biodistribution studies on rat model. J Drug Target. 2009;17(7):533-542.

12. Li D, Yang K, Li J-S, et al. Antitumor efficacy of a novel CLA-PTX microemulsion against brain tumors: in vitro and in vivo findings. Int J Nanomedicine. 2012;7:6105-6114.

13. Schleich N, Sibret P, Danhier P, et al. Dual anticancer drug/ superparamagnetic iron oxide-loaded PLGA-based nanoparticles for cancer therapy and magnetic resonance imaging. Int J Pharm. 2013; 447(1):94-101.

14. Schleich N, Po C, Jacobs D, et al. Comparison of active, passive and magnetic targeting to tumors of multifunctional paclitaxel/SPIO-loaded nanoparticles for tumor imaging and therapy. $J$ Control Release. 2014;194:82-91.

15. Freichels H, Danhier F, Préat V, Lecomte P, Jérôme C. Fluorescent labeling of degradable poly(lactide-co-glycolide) for cellular nanoparticles tracking in living cells. Int J Artif Organs. 2011;34(2):152-160.

16. Garinot M, Fiévez V, Pourcelle V, et al. PEGylated PLGA-based nanoparticles targeting M cells for oral vaccination. J Control Release. 2007;120(3):195-204.

17. Massart R. Preparation of aqueous magnetic liquids in alkaline and acidic media. IEEE Trans Magn. 1981;17(2):1247-1248.

18. Miyazawa T, Nakagawa K, Harigae T, et al. Distribution of $\beta$-caroteneencapsulated polysorbate 80-coated poly(D, L-lactide-co-glycolide) nanoparticles in rodent tissues following intravenous administration. Int J Nanomedicine. 2015;10:7223-7230.

19. Danhier P, De Preter G, Boutry S, et al. Electron paramagnetic resonance as a sensitive tool to assess the iron oxide content in cells for MRI cell labeling studies. Contrast Media Mol Imaging. 2012;7(3):302-307.

20. Danhier F, Pourcelle V, Marchand-Brynaert J, Jérôme C, Feron O, Préat V. Chapter eight - targeting of tumor endothelium by RGD-grafted PLGA-nanoparticles. In: Düzgüneş N, editor. Methods in Enzymology. Vol. 508. San Diego: Academic Press; 2012:157-175.

21. Danhier F, Messaoudi K, Lemaire L, Benoit J-P, Lagarce F. Combined anti-galectin-1 and anti-EGFR siRNA-loaded chitosan-lipid nanocapsules decrease temozolomide resistance in glioblastoma: in vivo evaluation. Int J Pharm. 2015;481(1):154-161.

22. Bianco J, Bastiancich C, Joudiou N, Gallez B, des Rieux A, Danhier F. Novel model of orthotopic U-87 MG glioblastoma resection in athymic nude mice. J Neurosci Methods. 2017;284:96-102.

23. Leten C, Struys T, Dresselaers T, Himmelreich U. In vivo and ex vivo assessment of the blood brain barrier integrity in different glioblastoma animal models. J Neurooncol. 2014;119(2):297-306.

24. Heye AK, Culling RD, Valdés Hernández MDC, Thrippleton MJ, Wardlaw JM. Assessment of blood-brain barrier disruption using dynamic contrast-enhanced MRI. A systematic review. Neuroimage Clin. 2014;6:262-274.

25. Wang B, Lv L, Wang Z, et al. Improved anti-glioblastoma efficacy by IL-13R $\alpha 2$ mediated copolymer nanoparticles loaded with paclitaxel. Sci Rep. 2015;5:16589.

26. Gu G, Xia H, Hu Q, et al. PEG-co-PCL nanoparticles modified with MMP-2/9 activatable low molecular weight protamine for enhanced targeted glioblastoma therapy. Biomaterials. 2013;34(1):196-208.

27. Bae YH, Park K. Targeted drug delivery to tumors: myths, reality and possibility. J Control Release. 2011;153(3):198-205. 
28. Mundargi RC, Babu VR, Rangaswamy V, Patel P, Aminabhavi TM. Nano/micro technologies for delivering macromolecular therapeutics using poly(D,L-lactide-co-glycolide) and its derivatives. J Control Release. 2008;125(3):193-209.

29. Jiang Y, Wang X, Liu X, et al. Enhanced antiglioma efficacy of ultrahigh loading capacity paclitaxel prodrug conjugate self-assembled targeted nanoparticles. ACS Appl Mater Interfaces. 2017;9(1): 211-217.

30. Zhu X-M, Wang Y-XJ, Leung KC-F, et al. Enhanced cellular uptake of aminosilane-coated superparamagnetic iron oxide nanoparticles in mammalian cell lines. Int J Nanomedicine. 2012;7:953-964.

31. Weaver BA. How Taxol/paclitaxel kills cancer cells. Mol Biol Cell. 2014;25(18):2677-2681.

32. Oh N, Park J-H. Endocytosis and exocytosis of nanoparticles in mammalian cells. Int J Nanomedicine. 2014;9(Suppl 1):51-63.

33. Yameen B, Choi WI, Vilos C, Swami A, Shi J, Farokhzad OC. Insight into nanoparticle cellular uptake and intracellular targeting. J Control Release. 2014;190:485-499.

34. Xu S, Olenyuk BZ, Okamoto CT, Hamm-Alvarez SF. Targeting receptor-mediated endocytotic pathways with nanoparticles: rationale and advances. Adv Drug Deliv Rev. 2013;65(1):121-138.

35. Saraiva C, Praça C, Ferreira R, Santos T, Ferreira L, Bernardino L. Nanoparticle-mediated brain drug delivery: overcoming blood-brain barrier to treat neurodegenerative diseases. J Control Release. 2016; 235:34- 47.

36. Lenting K, Verhaak R, ter Laan M, Wesseling P, Leenders W. Glioma: experimental models and reality. Acta Neuropathol. 2017;133(2): 263-282.

37. Gerstner ER, Fine RL. Increased permeability of the blood-brain barrier to chemotherapy in metastatic brain tumors: establishing a treatment paradigm. J Clin Oncol. 2007;25(16):2306-2312.

38. Zhang RD, Price JE, Fujimaki T, Bucana CD, Fidler IJ. Differential permeability of the blood-brain barrier in experimental brain metastases produced by human neoplasms implanted into nude mice. Am J Pathol. 1992;141(5):1115-1124.

39. Formolo CA, Williams R, Gordish-Dressman H, MacDonald TJ, Lee NH, Hathout Y. Secretome signature of invasive glioblastoma multiforme. J Proteome Res. 2011;10(7):3149-3159.

40. Claes A, Gambarota G, Hamans B, et al. Magnetic resonance imagingbased detection of glial brain tumors in mice after antiangiogenic treatment. Int J Cancer. 2008;122(9):1981-1986.
41. Dawidczyk CM, Russell LM, Searson PC. Perspective: recommendations for benchmarking pre-clinical studies of nanomedicines. Cancer Res. 2015;75(19):4016-4020.

42. Wang C-X, Huang L-S, Hou L-B, et al. Antitumor effects of polysorbate- 80 coated gemcitabine polybutylcyanoacrylate nanoparticles in vitro and its pharmacodynamics in vivo on C6 glioma cells of a brain tumor model. Brain Res. 2009;1261:91-99.

43. Gelperina S, Maksimenko O, Khalansky A, et al. Drug delivery to the brain using surfactant-coated poly(lactide-co-glycolide) nanoparticles: influence of the formulation parameters. Eur J Pharm Biopharm. 2010; 74(2):157-163.

44. Ishihara H, Kubota H, Lindberg RLP, et al. Endothelial cell barrier impairment induced by glioblastomas and transforming growth factor $\beta 2$ involves matrix metalloproteinases and tight junction proteins. J Neuropathol Exp Neurol. 2008;67(5):435-448.

45. Chertok B, Moffat BA, David AE, et al. Iron oxide nanoparticles as a drug delivery vehicle for MRI monitored magnetic targeting of brain tumors. Biomaterials. 2008;29(4):487-496.

46. Blanco E, Shen H, Ferrari M. Principles of nanoparticle design for overcoming biological barriers to drug delivery. Nat Biotechnol. 2015; 33(9):941-951.

47. Amoozgar Z, Yeo Y. Recent advances in stealth coating of nanoparticle drug delivery systems. Wiley Interdiscip Rev Nanomed Nanobiotechnol. 2012;4(2):219-233.

48. Gu L, Fang RH, Sailor MJ, Park J-H. In vivo clearance and toxicity of monodisperse iron oxide nanocrystals. ACS Nano. 2012;6(6): 4947-4954.

49. Wei Y, Zhao M, Yang F, Mao Y, Xie H, Zhou Q. Iron overload by superparamagnetic iron oxide nanoparticles is a high risk factor in cirrhosis by a systems toxicology assessment. Sci Rep. 2016;6:29110.

50. Garcia SC, Guterres SS, Bubols GB, Bulcão RP, Charão MF, Pohlmann AR. Polymeric nanoparticles: in vivo toxicological evaluation, cardiotoxicity, and hepatotoxicity. In: Durán N, Guterres SS, Alves OL, editors. Nanotoxicology: Materials, Methodologies, and Assessments. New York: Springer; 2014:299-324.

51. Jain TK, Reddy MK, Morales MA, Leslie-Pelecky DL, Labhasetwar V. Biodistribution, clearance, and biocompatibility of iron oxide magnetic nanoparticles in rats. Mol Pharm. 2008;5(2):316-327.

52. Danhier F. To exploit the tumor microenvironment: since the EPR effect fails in the clinic, what is the future of nanomedicine? J Control Release. 2016;244(Pt A):108-121. 


\section{Supplementary material}

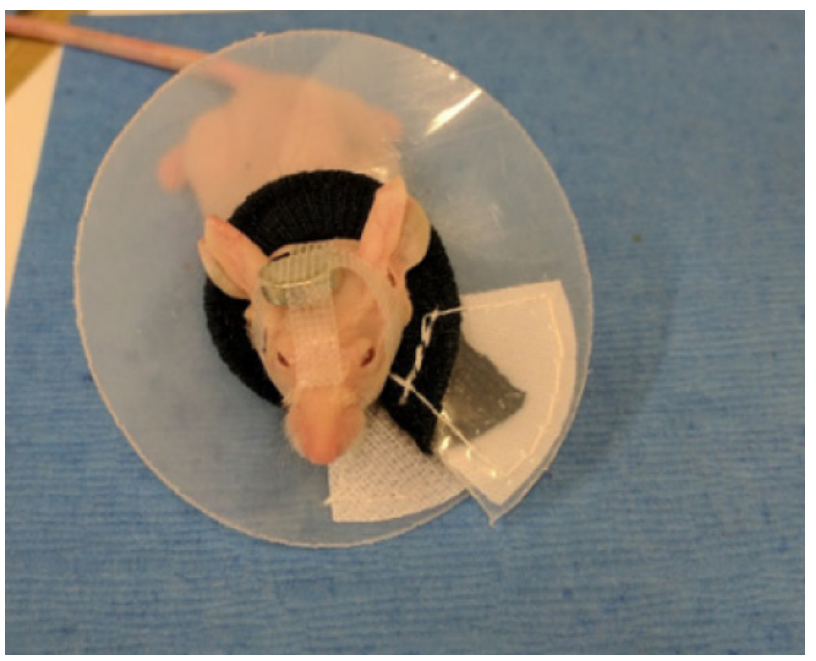

Figure SI NMRI nude mouse with neodymium magnet and Elizabethan collar. Abbreviation: NMRI, nuclear magnetic resonance imaging.

\section{Publish your work in this journal}

The International Journal of Nanomedicine is an international, peerreviewed journal focusing on the application of nanotechnology in diagnostics, therapeutics, and drug delivery systems throughou the biomedical field. This journal is indexed on PubMed Central,

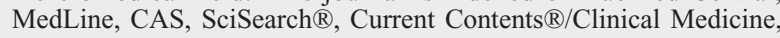

Journal Citation Reports/Science Edition, EMBase, Scopus and the Elsevier Bibliographic databases. The manuscript management system is completely online and includes a very quick and fair peer-review system, which is all easy to use. Visit http://www.dovepress.com/ testimonials.php to read real quotes from published authors.

\footnotetext{
Submit your manuscript here: http://www.dovepress.com/international-journal-of-nanomedicine-journal
} 